\title{
Delivering more than the "Sum of the Parts": using Quantitative Storytelling to address the challenges of conducting science for policy in the $E U$ land, water and energy nexus
}

\author{
K.B. Matthews ${ }^{\text {a, }}$ K.L. Blackstock ${ }^{\text {a }}$, M. Rivington a, K, Waylen a, D.G. Miller a, D. Wardell-Johnson a, \\ Z. Kovacic ${ }^{\text {b }}$, A. Renner ${ }^{\text {b }}$, M. Ripa ${ }^{\text {b }}$, M. Giampietro ${ }^{\text {b,c }}$ \\ ${ }^{a}$ The James Hutton Institute, Aberdeen, Scotland \\ ${ }^{b}$ Institut de Ciència i Tecnologia Ambientals, Universitat Autonoma de Barcelona, Catalunya \\ 'Institució Catalana de Recerca i Estudis Avançats (ICREA), Barcelona, Catalunya \\ Email: keith.matthews@,hutton.ac.uk
}

\begin{abstract}
This paper introduces an EU Horizon 2020 project (MAGIC) being conducted between 2016 and 2020 to better understand how EU water, food, energy and biodiversity policies are linked together and to EU climate and sustainability goals. The research is thus another instance of a nexus study in which various forms of research-derived and other knowledge are combined to evaluate and improve policy and other decisionmaking. The project is shaped by insights on the conduct and evaluation of inter- and transdisciplinary research for policy support reported through the MODSIM and iEMSs conferences in recent years. MAGIC brings together, from multiple centres, expertise in biophysical, computational, economic and social sciences underpinned by theories of transdisciplinary science-for-governance. These MAGIC consortium partners also have experience of treading the fine line between challenging policies (which may be defended by vested interests) and supporting processes of policy reform (whose impetus and legitimacy rely on generating communities of interest). The key challenges for MAGIC are whether this breadth and depth of expertise can be combined in ways that are theoretically rigorous, practical within the resources available and deliver more than the sum of its parts, that is, with discernible impact beyond the science-policy interface (i.e. outwith academia).
\end{abstract}

The integrative core of the project is Quantitative Story Telling (QST), a process of making quality tests of the narratives that underlie or justify key policy positions. The qualities of the policy narratives assessed are their feasibility (within biophysical limits), viability (within the existing institutional context) and desirability (reflecting distributional and acceptability issues); using uses reformulations of existing statistical datasets and simple empirical transformations. The analysis is framed using a societal metabolism metaphor. That is, it focuses on the funds of land and human time needed to create the flows of materials, energy and money that reproduce and maintain the identity of the system of interest (e.g. current patterns and trajectories of consumption). The analysis is conducted simultaneously across scales (geographical or classificatory) to highlight key externalisation effects and dependencies that may undermine long-term security.

This quantitative testing of narratives is preceded and shaped by critical choices made by both policy stakeholders and the research team. Which narratives should we focus on? How should we explore these with our social metabolism analysis? Different stakeholders and team members may all have different preferences and epistemological positions. Failure to acknowledge, and where possible resolve, such fundamental differences could limit potential for impact by ensuring that researchers and policy makers have no shared issue framing. For MAGIC, there is thus a strong focus on, and investment in, using social science methods to understand the actors, framing and institutional context within which the QST is conducted and any outputs are used. Deliberative and inclusive processes (undertaking the research in mixed-teams of researchers and stakeholders) are being used to legitimise the process of analysis and ensure that the outputs of QST are salient and credible. The paper concludes by reflecting on how well the expertise developed by the authors in conducting policy support translates from the particular context of Scotland into the structures, processes and procedures of the EU.

Keywords: Nexus, policy, Europe, science for governance, transdisciplinary 
Matthews et al., Delivering more than the "Sum of the Parts": using Quantitative Storytelling to address the challenges of conducting science for policy in the EU land, water and energy nexus.

\section{INTRODUCTION}

This paper introduces an EU Horizon 2020 project (MAGIC) being conducted between 2016-2020 to better understand how EU water, food, energy, waste and biodiversity policies are linked together and to EU climate and sustainability goals, framed in terms of the nexus concept (Cairns and Krzywoszynska, 2016). This paper presents the political and scientific context that has shaped the project and then briefly sets out the overall structures of the project and how these are intended to support transdisciplinary research with policy makers. This interfacing of science and policy uses an approach termed Quantitative Story Telling (QST). QST uses quantitative, multi-scale assessments of changes in the funds and flows of land, human time, materials, energy and money to assess the narratives being used by stakeholders to justify either the status quo or alternative policy positions for the EU. The process of QST draws philosophically on post-normal science for governance. Here the quality of analytical outcomes depends on clarifying the choices that have shaped the content of the "evidence base" and the modes of analysis considered salient and credible. The QST is not therefore concerned with refinement of the minutiae of evidence but rather questioning whether existing science-policy consensuses are ignoring existential threats because they take too narrow view of the challenges faced by the EU.

\subsection{Political context}

The European Union continues to grapple with the long-standing challenges of balancing quality of life for its citizens, ensuring financial stability and safeguarding or enhancing environmental quality (air and water quality, soil and biodiversity loss and GHG emissions). The circle is squared, at least rhetorically, through the promotion of innovation, green growth and the bio- or circular economy. Yet the EU is faced by a rising tide of introspective populism epitomized by Brexit. This questions the value of the EU principles of collective action, coordination and integration and the wisdom of maintaining a Common Agriculture Policy at $40 \%$ of budget in 2015. The EU also has to confront a series on emerging challenges that have the potential to undermine further the perceived legitimacy of EU institutions (Spratt, et al. 2017). There is a demographic challenge with aging and often declining populations simultaneously dependent on migrant labour in the care system yet seeing such labour as undermining wages and the wider socio-cultural fabric. A security challenge is most apparent in the energy sector with the dependence of the EU on sources controlled by unstable or despotic regimes. For water security, there is the challenge of a changing climate that sees an increasing dependence on fossil water or desalination in the south of the EU. For food security, the certainties of relative scarcity and post-colonial control of resources via market and politico-military manipulation are slipping away. Finally, there are the challenges of seemingly perpetual austerity, increasing inequalities and structural deficits hugely exacerbated by the need to bail out failing casino-capitalist financial institutions. Minimum welfare and public service standards are thereby eroded, living standards visibly stagnate or decline and intergenerational tensions increase with new workers worse off than pensioners for the first time. In this context, there is a pressing need to re-examine narratives that have shaped policy to date and how these may need to be revised to reflect emerging realities.

\subsection{Science Context - can nexus studies be more than the new sustainability?}

The nature of the research commissioned recognises these policy challenges but also that the conduct of science itself is facing significant challenge (Saltelli and Giampietro 2015). There is an evident need for communitybuilding to transcend mechanistic scientist-policy maker separation and to take full advantage of the spectrum of actors and institutions encompassed by the nexus (Funtowicz and Strand 2007). In undertaking nexus studies there is the need to build and learn from the successes and failures of sustainability science in terms of its influence on policy. In particular, there is the need to avoid hypocognition since as noted by Stiglitz (2011) "Models by their nature are like blinders. In leaving out certain things, they focus our attention on other things. They provide a frame through which we see the world". What is needed are approaches that tackle not only the complicatedness of the nexus but also its complexity. This is achieved by checking (i) the robustness of the story-telling determining the pre-analytical choices - i.e. issue definition and problem structuring and (ii) integrating across scales and dimensions the non-equivalent quantitative assessments that can only be obtained by adopting a single dimension and scale of analysis at the time. This makes it possible to check carefully the assumptions of established models and to integrate analyses in a non-conventional way (see Giampietro et al. 2009).

\section{THE MAGIC PROJECT}

Against this background, the MAGIC project brings together, from multiple research centres across the EU, expertise in biophysical, computational, economic and social sciences underpinned by theories of transdisciplinary science-for-governance. The quantitative engine of MAGIC is MuSIASEM (Multi-Scale 
Matthews et al., Delivering more than the "Sum of the Parts": using Quantitative Storytelling to address the challenges of conducting science for policy in the EU land, water and energy nexus.

Integrated Analysis of Societal and Ecosystem Metabolism) an innovative method of accounting having the goal of keeping coherence across scales and dimensions of quantitative assessments generated using different metrics. This has been applied in a variety of settings including nexus studies (see Giampietro et al. 2014). MuSIASEM analysis is framed using a societal metabolism metaphor. That is, it focuses on the funds of land and human time needed to create the flows of materials, energy and money that reproduce and maintain the identity of the system of interest (e.g. current patterns and trajectories of consumption). The analysis is conducted simultaneously across scales (geographical or hierarchical/functional) to highlight contrasts and key externalisation effects and dependencies that may undermine long-term security. The key challenge for MAGIC is whether this breadth and depth of expertise can be combined in ways that are theoretically rigorous, practical within the resources available and deliver more than the sum of its parts, that is, with discernible impact beyond the science-policy interface.

\subsection{Expectation of impact}

In common with other large-scale EU H2020 projects the statement of scope was broad ranging, reflecting the involvement of multiple actors in shaping the call text. The scope suggested tool development, methodologies for integration and modelling approaches but also raised questions of conceptual understanding, scales of analysis, implications for policy and regulation and barriers to implementation. Yet while the expectations of what and how of the project would deliver were unclear, the statement on the expectation of impact was very specific and served as the basis from which the MAGIC project was defined:

- Increased understanding of how: water management, food and biodiversity policies are linked together and to climate and sustainability goals.

- Reduction of the uncertainties about the opportunities and limitations of low-carbon options, such as bioenergy technologies and resource efficiency measures, in view of relevant near-term policy initiatives.

- Contribution to future assessments, including those of the IPCC, with multidisciplinary and integrated tools.

\subsection{MAGIC structures and processes}

With the impact expectation in mind, the project was structured to support two processes of QST, one with a policy focus and another with an innovation/technology focus. There is of course recognition that there is potential for interaction between these two QST processes especially where policy has sought to promote particular technologies or innovations, which have implications for other aspects of the nexus (e.g. the use of biofuels). The project is structured as a set of seven work-packages (WPs); i.e. interdependent sub-projects that interact to generate the impacts sought. The interaction with policy and other stakeholders is mainly via two WPs - the Quality check on Policy Narratives (WP5, further elaborated in Section 3.2) and the Quality Check on Innovation Narratives (WP6). These two WPs are supported by four others with their roles indicated in Figure $\mathbf{1}^{1}$ and discussed below.

The Nexus Dialogue Space (WP2), which seeks to support the conduct of QST by providing expertise, facilities and tools to support the science-stakeholder interactions. For WP5 this is

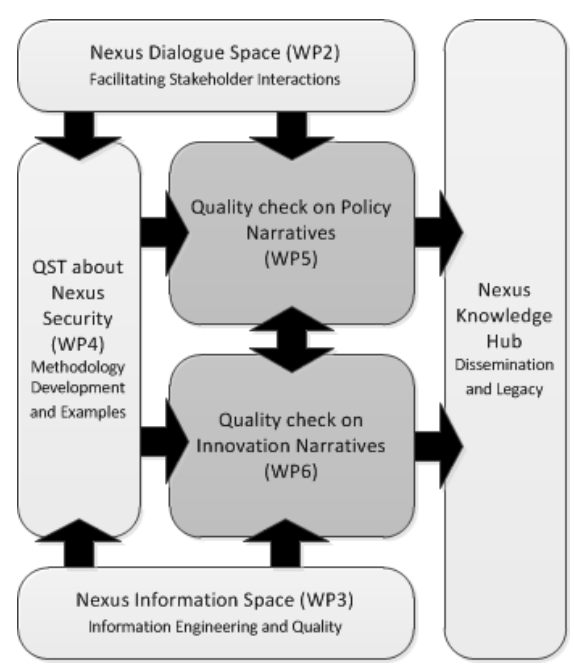

Figure 1. Component work packages of MAGIC about understanding and navigating through the formal and informal institutions of the EU (e.g. the Directorates General (DGs), Inter-service Groups, regulatory review, audit and evaluation bodies and the Parliamentary Committees). For WP6 the focus is different with the wider societal acceptability of technologies having a greater relevance (e.g. GM crops).

The Nexus Information Space (WP3), is developing tools and protocols to formalize the implementation of the MuSIASEM societal metabolism analysis. This includes both the engineering of decision support tools and open source databases organising data previously held and used in a variety of ad hoc formats. The WP is also developing interfaces to online data sources (e.g. EUROSTAT), protocols to track data pedigrees though

\footnotetext{
${ }^{1}$ The management, reporting and evaluation of progress processes for the project are dealt with in a dedicated management WP is omitted from the figure.
} 
Matthews et al., Delivering more than the "Sum of the Parts": using Quantitative Storytelling to address the challenges of conducting science for policy in the EU land, water and energy nexus.

the analysis process and to improve processes of reclassification and clustering (a common issue in dealing with the integration of multiple datasets across EU Member State jurisdictions).

The Nexus Knowledge Hub (WP7) handles dissemination to a variety of audiences where the intention is awareness raising and archiving of project outputs.

The QST about Nexus Security (WP4) is where development of QST takes place within the consortium. The examples developed here have key functions in the development and training of research teams across participating organizations, the creation of examples that can serve as the basis for discussion with stakeholders in the initial phases of WP's 5 and 6 and serve as a source of prototypes for customization later in the project. Having outlined the overall project organization, the following section elaborates QST in theory and as practiced in MAGIC.

\section{QUANTITATIVE STORY TELLING}

\subsection{QST as science for governance}

QST recognizes that there is unavoidable scientific uncertainty and value plurality in decision making within the nexus of water, energy and food policies. By providing an alternative set of tools and processes, QST seeks to promote progressive thinking about nexus/sustainability issues by:

1. Recognising the "evidence trap" whereby an absence of scientific certainty is used as a justification for inaction (which of course is, in effect, action in favour of existing beneficiaries of the sociopolitical status quo).

2. Recognizing that faux consensus (where stakeholders agree on headline objectives but with entirely different underlying interpretations) is less useful than articulating the nature of the conflicts; while realizing that it is not the role of science to resolve such conflicts and indeed to do so is likely illegitimate scientism in service of a particular interest group.

3. Recognizing that value plurality permeates nexus issues, shaping both what is considered evidence and how that evidence is interpreted. This is especially important for views on the fairness of burden sharing implied by the trade-offs inherent in wicked nexus issues.

Taken together this means that the semantic aspects of science for policy in QST necessarily have equal or greater weight compared with the formal (quantitative analysis or modelling) in determining the conduct of the process (see the stages of QST in Figure 2).

\subsection{The high-level QST stages}

Each of the QST stages is undertaken in Mixed Teams that include policy stakeholders from within the EU institutions working with researchers having both policy and domain knowledge (e.g. land use, water, biodiversity), and backgrounds in biophysical, social and computational sciences, coordinated by staff with experience of working across the science policy interface.

1. Summarizing the Narratives - this draws on materials from work in WP2 and identifies the narratives which are of interest to policy makers and other stakeholders. The phase will also assess which narrative are compatible with MuSIASEM analysis and see further interactions with policy and other stakeholders in building Mixed Teams for the specific domains.

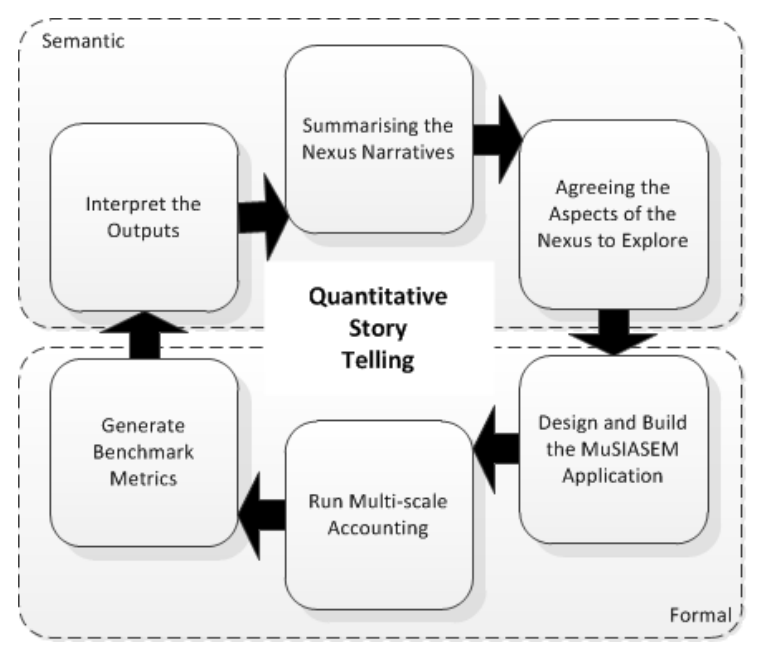

Figure 2. The process of Quantitative Story Telling

2. Agreeing Aspects to Explore - this stage sees the development of a much more specific shared understanding of what will be analysed and how (i.e. building a grammar). This stage moves progressively from deciding higher-level priorities (e.g. the type and numbers of narratives to be analysed), towards decisions on the specific aspects of systems that have to be represented (semantic definitions) and other pre-analytical choices that will shape the later quantitative analysis (e.g. setting system boundaries, scales of analysis, functional and structural types, and useful indicators). This will draw on examples from WP4. 
Matthews et al., Delivering more than the "Sum of the Parts": using Quantitative Storytelling to address the challenges of conducting science for policy in the EU land, water and energy nexus.

3. Design and Build the MuSIASEM Application - this stage sees the formalization (representation in the forms used in the process of analysis) of the systems that the narrative(s) address (e.g. a water body, an agrifood supply chain or an electricity-grid). This stage builds an analytical framework that while it represents a specific type of system is at least partially reusable for any instances of that type (see the next phase). This stage will build on and reuse tools and data from WP4 and other previous MuSIASEM applications and will later make use of the software being developed to support QST in WP3 the Nexus Information System.

4. Run Multi-Scale Analysis - in this stage the MuSIASEM Application from stage three is populated with quantitative data such that it becomes a complete formal representation of the system of interest and can be used to test narratives about the current state (diagnostic mode) and possible alternatives (simulation mode). The expectation is that this process will also be supported by developments in WP3 of the Nexus Information System.

5. Generate Benchmark Metrics - In this stage, summary metrics are generated that assess the feasibility (within biophysical limits), viability (within institutional limits - e.g. socio-economic limits) and desirability (distributional, burden sharing and other outcomes) of the narratives. The process of summarizing and communicating the outputs will need to be undertaken in close partnership with policy stakeholders within the Mixed Teams, and must convey both quantitative and qualitative (semantic) uncertainties and sensitivities. This is essential as the outputs from the analysis need to be salient and credible if they are to be influential in conceptual or instrumental terms.

6. Interpret the Outputs - this stage sees the deliberation on and interpretation of the significance of the outputs of the QST analysis with stakeholders and the shaping of any further stages - either with new narratives or with alternative cases.

While this process is necessarily presented in the text here as a linear process (even if iterative)-it is anticipated that there may well be occasions for some Mixed Teams, where insights gained within the QST process mean that it is desirable to return to earlier QST stages and to modify or refine the analysis. The intention is not, however, to pursue ever-greater depth of partial analysis but to complete the QST cycle and generate meaningful outputs that stimulate deliberations with stakeholders. This will better demonstrate the potential of QST and MuSIASEM, highlight challenges of the use of QST in practice (e.g. data availability) and build credibility with stakeholders so that there is longer lasting positive engagement. At present, it is proposed to focus further iterations more specifically on the challenges of policy cohesion and the delivery of EU Sustainability and Climate Change goals.

\section{DISCUSSION: MORE THAN THE SUM OF THE PARTS?}

This section reflects on progress in the first year of the MAGIC project. In particular, it questions how well methods and experience developed in small, tightly knit, interdisciplinary research teams can be translated into a larger multi-centre consortium working in a new policy context.

\subsection{Epistemological readiness - buying into transdisciplinary working}

The MAGIC project makes significant and specific demands on researchers that they may well not have faced in more conventionally framed academic research. MAGIC as a whole, and especially WP5 ('the Quality check of policy narratives') have been shaped by the long experience of the authors in diverse fields of endeavour as discussed in MODSIM and iEMSs conferences since 2003. The common thread has been to seek to more effectively engage across the science-stakeholder interface (however conceptualised, e.g. Matthews et al 2015). The expectations in MAGIC include embracing interdisciplinary working, not merely resolving ontological differences but also the deeply ingrained epistemic differences that fundamentally shape what is research and thus what counts as evidence. The further expectation of transdisciplinary working requires another phase-shift of lexicons, priorities and timescale and the need for a deep appreciation for how science interacts with policy. The change to transdisciplinary working, that has taken years for the authors, is being asked of other partners in weeks or months. Questions remain of how easy such transitions can be for staff from organisations without strong institutional commitment to transdisciplinary working, or at stages of their careers when conventional measures of academic esteem or impact are of greater importance.

\subsection{Scale - when is bigger better?}

The sheer size of an H2020 consortium is both a blessing and a curse for this kind of project where deep integration and peer-to-peer learning is an expectation (rather than continuing business-as-usual research with an additional $\mathrm{PhD}$ student or two). The resulting benefit is in the availability of a depth of expertise that goes 
Matthews et al., Delivering more than the "Sum of the Parts": using Quantitative Storytelling to address the challenges of conducting science for policy in the EU land, water and energy nexus.

well beyond that which can be generated within any single organisation. Yet the harnessing of such expertise represents a coordination overhead that challenges research leaders' capabilities. For example, there are five mixed teams for the policy QST work package, staffed from all nine partners, that need to be coordinated such that they deliver both impact with their respective stakeholders and insights that are sufficiently comparable to form the basis of later studies between policy domains. There are particular challenges in integrating researchers from institutions with strong disciplinary expertise but little experience of integrating natural and social science or in interacting with policy makers. To be successful the consortium leadership needs to generate and exploit bonding and bridging social capital and to do so within tight time constraints and across multiple sites. Particular challenges are also evident when leadership roles have nominal time commitments and when delivery falls on inexperienced staff recruited specifically to work on the project.

Another scaling challenge is raised by the need for substantial elements of underpinning technical development. The intention here is to simplify the process of using the MuSIASEM methods by providing an infrastructure of data, tools and protocols and to thus allow a wider range of researchers to make use of the methods. To do this requires skillsets and expertise that are beyond those included in previous incarnations of MuSIASEM, for example software engineering, data curation and statistical analyses. There is, however, an inherent tension within consortia between the utilitarian needs of stakeholder-facing teams and the need for technical and methodological development to be innovation led, when research teams deliver them. On the other hand, it is unlikely that conventional IT service providers would find attractive the open-ended process of coconstruction. The compromise is in generating novel but reusable tools (grammars, data structures, data sources etc.). While this can increase efficiency long term, there is always the danger of such infrastructures making the problem fit the solution and with the timeliness of their availability.

\subsection{Combining qualitative and quantitative analysis}

MAGIC is particularly ambitious in the degree to which it seeks to embed both the qualitative and quantitative methods within its research processes with the intention that they are mutually enriching. While this ambition is born of clear theoretical insight, the practical implementation that meshes such differing entities remains particularly challenging especially with the number of parallel instances of policy-led analysis envisaged within MAGIC. Two pathologies of mixing qual-quant methods are apparent from the authors' previous experiences. These can be caricatured by qualitative researchers always responding to any query "It depends" and quantitative researchers responding, "We can't model that". Resolving these differences depends largely on compromise on the part of qualitative researchers in recognising the need for some degree of generalisation and quantitative researchers recognising that, however well-crafted, a model of the wrong thing is useless. In a multi-centre, complex research project, it is essential that sufficient resource be built in for interdisciplinary team building and the generation of cross-centre cooperation.

A remaining challenge, however, is in meshing time lines between the qualitative led, and semantically dominated processes and the quantification led and formalisation (or modelling) dominated processes. This is especially challenging when new formalisation processes are being developed in parallel with the engagement of policy stakeholders. New formalisations can take a substantial time to create and this makes it difficult to maintain an active engagement with stakeholders participating in the co-construction of the research. In MAGIC, this was anticipated and the intention has been to mitigate this by drawing on a repository of existing formalisms. Yet even here, the danger is their availability shapes the analysis rather than the other way round. It remains an open question whether beyond the scope of a research project it will be possible to deploy the approach and the tools within the time constraints of a policy cycle.

\subsection{Institutional insights: finding a niche}

Interactions with Directorates General (DG) staff and others have taken place in a variety of forums (interviews in WP2, workshops and one-to-one meetings). These interactions have provided a key insight that is reshaping the anticipated conduct of the Quality check for Policy almost immediately: the benefit of liaising across DGs. Within the proposal, the intention was that the initial engagement would be with staff of the individual DG's with primary responsibility for particular directives or policies. The expectation was that through a process of awareness raising and co-construction it would be possible to ensure that the analyses being carried out were salient and to start to bootstrap credibility in a similar way to the policy-led analysis undertaken with Scottish Government. We anticipated that the second phase would address policy coherence across the Directorates. There is, however, an opportunity to revise the plan and to start with cross-DG working. From the interviews with EU staff there is an institutional desire to see progress in cross-DG working with existing examples of new practices and revisions of structures within DGs intended to open up internal silos. Within the DGs, staff interacting with MAGIC were also more receptive to radical alternatives than was anticipated. There is an 
Matthews et al., Delivering more than the "Sum of the Parts": using Quantitative Storytelling to address the challenges of conducting science for policy in the EU land, water and energy nexus.

appetite for a multi-perspective assessment of policy options, which identifies the nature of trade-offs or constraints and which challenges currently dominant narratives. Their advice is that well-established and strongly credible incumbents do not already fill the niche for such analysis, as is the case for any within DG working. Avoiding unnecessary competition with incumbent experts has long been known as a key factor increasing the likelihood of success in decision support (Matthews et al 2007). This insight is a motivation to reshape MAGIC in the next year to explore the new options but the challenge is how to redirect five Mixed Teams with existing research agendas.

\section{CONCLUSIONS}

The structures and processes of the MAGIC project reflect the bringing together of several strands of theory and practice. The individual partners each have strong reputations within their respective fields and most have experience in working in EU consortium based research. The fund of knowledge and experience though needs to be translated into flows of impact. Does MAGIC really have the potential to deliver more than the sum of the parts? More academic partners can generate new capability by peer-to-peer learning and are more likely to mean enduring connectivity. The profile of larger projects also increases their potential at least to generate initial interest with policy stakeholders. It is uncertain though how well larger projects function in generating change in working cultures or conceptual change given the likely resistance to this from the partners home institutions. The overheads of inter- and transdisciplinary working are also compounded by the need to coordinate activity across sites and sub-projects. Larger projects are inevitably less agile and potentially more fragile where there are interdependencies and this may seriously impair their ability to deliver instrumental change within the time lines of policy processes. To deliver more than the sum of the parts the emphasis has to be on capitalising on positive emergent (or unanticipated) phenomena from the interactions between the planned components and adapting to reinforce success.

\section{ACKNOWLEDGMENTS}

This project was funded by the European Union's Horizon 2020 research and innovation programme (Grant agreement No 689669). This work reflects only the author's view and the funding agency is not responsible for any use that may be made of the information it contains. The James Hutton Institute is supported by the Scottish Government's Rural and Environment Science and Analytical Services Division (RESAS)

\section{REFERENCES}

Cairns, R. and Krzywoszynska (2016). Anatomy of a buzzword: The emergence of 'the water-energy-food nexus' in UK natural resource debates, Environmental Science \& Policy, 64, 164-170.

Funtowicz, S. and Strand, R. (2007). Models of Science and Policy, in Traavik, T. and Lim, L.C. (eds.) Biosafety First: Holistic Approaches to Risk and Uncertainty in Genetic Engineering and Genetically Modified Organisms, Trondheim: Tapir, pp 263-278.

Giampietro, M., Mayumi, K. And Ramos-Martin, J. (2009). Multi-scale integrated analysis of societal and ecosystem metabolism (MuSIASEM): Theoretical concepts and basic rationale. Energy 34, 313-322.

Giampietro, M., Aspinall, R., Ramos-Martin, J. and Bukkens, S.G.F. (Eds.) (2014). Resource Accounting for Sustainability: The Nexus Between Energy, Food, Water and Land Use. Routledge.

Matthews, K. B., Rivington, M., Blackstock, K. L., McCrum, G., Buchan, K., and Miller, D. G. (2011). Raising the bar? - The challenges of evaluating the outcomes of environmental modelling and software, Environmental Modelling and Software 26(3), 247-257.

Matthews, K. B., Schwarz, G., Buchan, K., Rivington, M., and Miller, D. G. (2007). Wither agricultural DSS?, Computers and Electronics in Agriculture 61, 149-159.

Matthews, K.B., Miller, D.G. and Wardell-Johnson, D. (2015). Practicing and evaluating outcomes of working across the science policy interface, In Weber, T., McPhee, M.J. and Anderssen, R.S. (eds) MODSIM2015.

Saltelli, A. and Giampietro, M. (2017). What is wrong with evidence based policy, and how can it be improved? Futures 91, 62-71, doi.org/10.1016/j.futures.2016.11.012.

Spratt, S., Simms, A., Neitzert, E. and Ryan-Collins, J. (2017). The Great Transition: A tale of how it turned out right, Part of Finding ways to survive and thrive through financial and climate crises and the peak and decline of global oil production. New Economics Foundation (online).

Stiglitz, J. (2011). Rethinking macroeconomics: what failed, and how to repair it, Journal of the European Economic Association, Vol. 9, No. 4, pp.591-645. 